\title{
Breve cartografía de una disonancia epistémica: educación, complejidad y reforma*
}

\author{
Iván Oliva Figueroa**
}

\begin{abstract}
Resumen: En el artículo se desarrollan algunas ideas asociadas al campo de la epistemología, ciencias cognitivas y ciencias de la educación desde una aproximación compleja. En este contexto, la noción de disonancia epistémica refiere a una interpretación crítica de algunos de los axiomas paradigmáticos que subyacen y legitiman, en sus diversos niveles y dimensiones, los modelos de desarrollo en la educación contemporánea.
\end{abstract}

Palabras clave: epistemología, complejidad, educación

\section{Brief cartography of an epistemic dissonance: education, complexity and reform}

\begin{abstract}
Some of the ideas developed in this paper are related to the different fields associated to epistemology, cognitive and educational sciences, all of them observed from a complex perspective. In this context, the notion of epistemic dissonance refers to a critical interpretation of some of the paradigmatic axioms which underlie and legitimize, through their different levels and dimensions, the developmental models in contemporary education.
\end{abstract}

Key words: epistemology, complexity, education

\section{Breve cartografía de uma disonancia epistémica: educação, complexidade e reforma}

Resumo: No artigo desenvolvem-se algumas ideias associadas ao campo da epistemología, ciências cognitivas e ciências da educação desde uma aproximação complexa. Neste contexto, a noção de disonancia epistémica refere a uma interpretação crítica de alguns dos axiomas paradigmáticos que subyacen e legitiman, em seus diversos níveis e dimensões, os modelos de desenvolvimento na educação contemporânea.

\footnotetext{
* El presente trabajo se desprende de un seminario de trabajo realizado durante octubre de 2008 en la ciudad de la Serena en el contexto del Proyecto FONDECYT Regular 1080073 “Complejidades educativas emergentes y caóticas en la escuela lineal” de responsabilidad del investigador Dr. Carlos Calvo Muñoz. Asimismo, los tópicos desarrollados responden al trabajo teórico preliminar del Proyecto FONDECYT Iniciación 11080193 "Conocimiento, complejidad y transdisciplina: Un estudio paradigmatológico en Universidades chilenas" de responsabilidad del autor del presente documento.

** Universidad Austral de Chile, Valdivia, Chile. Email: ivanoliva@uach.cl
} 
Palavras-chave: epistemologia, complexidade, educação

Recibido: 23.03.2009

Aceptado: 27.05.2009

$* * *$

La interpretación de la sociedad contemporánea ha entretejido, sostenido y estabilizado sus explicaciones utilizando la hebra cognitiva y, con ello, ha transformando la noción de conocimiento en uno de los axiomas más importantes de sus modelos explicativos. Sociedad del conocimiento, sociedad de la información, economías basadas en el conocimiento, sociedad de la incertidumbre, gestión del conocimiento, aprendizaje organizacional, entre otras, proliferan en los procesos explicativos a nivel global. Propuestas basadas en la idea de duplicación de conocimiento cada intervalos de tiempo cada vez menores, o su variante, la depreciación del conocimiento a intervalos cada vez más pequeños de tiempo, son falacias que arriesgan situar un proceso sistémico multidimensional, en un dominio de interpretación que remite a progresos lineales y sumativos.

Pese a su centralidad explicativa, el fenómeno del conocer ha sido mutilado y unidimensionalizado, predominando una concepción acumulativa, bancaria y objetual en su interpretación. En este sentido, el conocimiento y sus nociones íntimamente asociadas, parecen explicar mucho, pero en definitiva, poco las explica a ellas. Esto último comporta riesgos epistemológicos serios para las concepciones de desarrollo humano y social que devienen de estas interpretaciones y que se distribuyen holográficamente ${ }^{1}$ desde sus procesos educativos.

En consecuencia, parte importante de las problemáticas educativas contemporáneas pueden pasar por una restringida y unidimensional concepción del fenómeno del conocer, configurando un nodo crítico no sólo a nivel de político, sino además a un meta-nivel epistemológico. Una reformulación paradigmática de algunos dominios de interpretación del fenómeno del conocer, pueden contribuir a resituar los fundamentos epistemológicos, desde los cuales se ha configurado el sentido del fenómeno educativo $\mathrm{y}$, con ello, abordar la pertinencia de sus dimensiones de desarrollo. Una reforma educativa (y con ello del conocer) que aspire a una inflexión epistemológica, desbordará cualquier ponderación netamente estructural, al centrar su interés en las regiones cualitativas y complejas del conocimiento y no sólo en aspectos triviales, sumativos o de proyección lineal. Las formas de interpretación del fenómeno del conocer infiltran y co-generan los esquemas de acción en educación con alcances en todos sus niveles, es por ello que nunca son inocentes.

\footnotetext{
${ }^{1}$ La idea holográfica hace referencia a la presencia fenomenológica del todo en la parte y la parte en el todo.
} 


\section{Desde la acumulación a la configuración}

Las intoxicaciones causadas por la instrucción son mucho más graves que las intoxicaciones de los subproductos de la industria

\section{R. Ruyer}

Es quizás, la trágica premisa de Lord Kelvin: “Todo cuanto existe, existe en una cantidad y por ende puede ser medido", la que subyace a la enajenación en la sumatoria, acumulación y medición de conocimiento en las sociedades contemporáneas. No obstante, la pertinencia y ética del conocer humano no se expresa en la acumulación sino en su organización y metacontextualización; en otras palabras, en campos de asociatividad no lineales y pautas de vínculos cognitivos. En este sentido, más que medir, acumular y depreciar conocimiento, es necesario cartografiar sus patrones y sus dimensiones de pertinencia y contextualización, transitando desde una lógica elemental referida a componentes y unidades cognitivas (datos, información adimensional) a una configuracional (referida a formas, pautas y contextos semióticos), tal como esquematiza la siguiente figura.

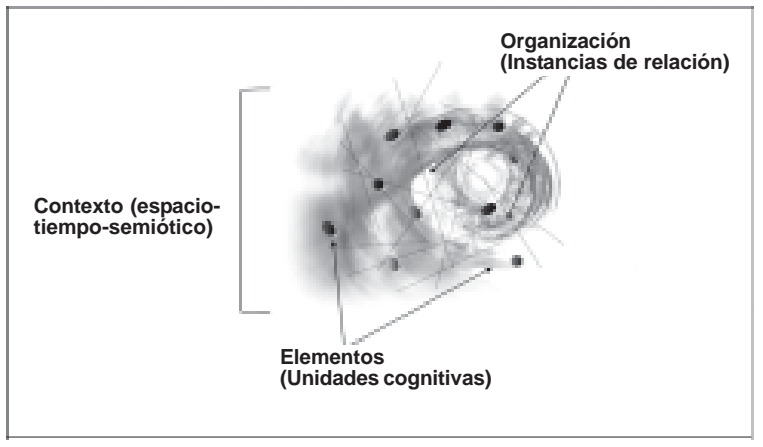

La organización del conocer humano es una tarea a la vez capital, aleatoria e incierta; para ello hay que comprender que la revolución se juega hoy no en la oposición ni acumulación, sino en el terreno de la complejidad del modo de organización de las ideas, lo que requiere pensar lo educativo de forma radicalmente compleja (Morin, 1992). Es este dominio cualitativo del conocimiento el que posee mayores implicancias en lo referido al desarrollo multidimensional en la denominada economía del conocimiento, dado que es en este ámbito de la reflexión donde está la posibilidad de pensar la flexibilidad, creatividad y complejidad, como uno de los mayores recursos de desarrollo de la cognición humana.

En este sentido, la política pública en educación más que enfatizar en el concepto de brecha digital, debería abordar el entendimiento de la brecha analógica del conocer, la cual ni siquiera en rigor es una brecha (las cuales sólo son posibles de postular entre dos puntos determinados en un espacio), sino más bien una disonancia epistémica. Las problemáticas 
educativas no están situadas en un espacio cartesiano y sus problemáticas no son las distancias espaciales o temporales, sino las pautas, integración y contextualización de los patrones de conocimiento co-creados por sus participantes. Cuando le expresamos a otra persona: "te siento distante”, no nos referimos a un problema de distancia (brecha) en el espacio o tiempo, sino que evocamos disonancias o no-equivalencias en nuestras formas afectivas-lógicas de configurar mundo, lo que a mi modo de entender es un problema analógico y no digital, cognitivo y no espacial, complejo y no lineal.

El progreso del conocimiento humano no se lleva a cabo en instancias binarias, digitales o acumulativas, sino en instancias analógicas, asociativas y meta-asociativas, que han sido pobremente tratadas en el contexto educativo formal. Transferir, depositar, recibir, reproducir conocimiento, entre otras, abundan en los modelos explicativos contemporáneos en torno a las sociedades basadas en el conocimiento y también en la teoría educativa; no obstante, estas acciones sólo son posibles de ejercer a sustancias y no a procesos. Tal como advierte von Foerster (1996:189) "No es de extrañar que un sistema educacional que confunde el proceso de crear nuevos procesos [de distinciones] ${ }^{2}$ con la distribución de mercancías llamadas “conocimiento” puede causar alguna insatisfacción en los hipotéticos receptores, porque las mercancías no llegan: no hay tales mercancías.” El concepto de educación bancaria denunciado por Freire, no es sólo un problema ético y político de la educación, sino que además comporta una imposibilidad fisiológica en términos de cómo opera la vida y la cognición, lo que se traduce en profundas falacias epistemológicas en todos los niveles de descripción del fenómeno.

Con lo anterior nos alejamos de perspectivas de orden lineal (tales como la relación emisor à receptor) en torno a la interpretación de la comunicación, el aprendizaje y la educación, para situarlos en una dimensión sistémica y recursiva de la cognición. De este modo, la transmisión del conocimiento en este proceso no es posible, dado que aceptamos la autonomía cognitiva del sujeto y el carácter sistémico de los procesos de conocimiento. Es por ello que la interpretación del fenómeno educativo no puede confundir sustancias cognitivas (susceptibles de transferir, medir y pesar) con procesos no lineales y distribuidos. Derivado de lo anterior, se da un tránsito cognitivo desde la secuencialidad, la linealidad y el subyacente determinismo, a la simultaneidad, convergencia y emergencia configuracional/co-participativa de sentido.

Abordar el conocimiento desde un enfoque analógico y configuracional es, a la vez, abordarlo en su dimensión paradigmática, enfatizando en sus instancias de separación, asociación, jerarquización y

${ }^{2}$ En este contexto, un acto de distinción es la generación de una diferencia o contraste básico que devendrá en unidades para un sistema cognitivo. En consecuencia, toda observación involucra por parte de un sistema cognitivo, aplicaciones de esquemas de diferencias que le permiten generar un mundo cognitivo. 
centralización; esto es, en los principios supralógicos de organización del conocer. Para Morin (1994) esto es congruente con la noción de paradigma en dos dimensiones: primero, un paradigma modela la restricción/selección de los conceptos neurálgicos de la inteligibilidad. Segundo, condiciona las operaciones lógicas maestras en los sistemas de observadores.

Estos principios orientan y pautan los procesos de configuración de sentido, cohesionando la trama de distinciones al interior de una comunidad de observadores ${ }^{3}$. Estos procesos, al accionar recursivamente en los espacios comunicacionales educativos, pueden tender a la latencia, arriesgando cegueras epistemológicas fundamentales. Insistamos, desde una dimensionalidad compleja, el fenómeno del conocer no puede situarse únicamente bajo un prisma unidimensional, sumativo y objetual, ya que esto supone un reduccionismo y trivialización que no aborda dominios fundamentales del conocimiento tales como: a) la forma u organización paradigmática de determinadas instancias cognitivas, b) las redes comunicacionales emergentes productoras y productos de a), y c) las implicancias éticas de estos procesos configuracionales.

\title{
Desde la descripción a la generación de mundos cognitivos
}

\author{
El problema no es el mundo; el problema lo crea uno \\ en su relación con el otro, \\ y ese problema, al extenderse, \\ se convierte en el problema del mundo.
}

Krishnamurti.

El énfasis representacionalista y descriptivo de la cognición tiene quizás, uno de sus correlatos históricos más relevantes en la división realizada por Descartes en el S.XVII, entre res cogitans (sustancia pensante) y res extensa (sustancia extensa). Esta desintegración del fenómeno cognitivo y su consecuente sustantivación, involucró una noción de conocimiento basada en la intervención de sustancias disociadas y no de procesos autoeco organizados.

A la luz de las ciencias cognitivas contemporáneas, fenomenología y epistemología de la complejidad, la noción de sujeto recupera su dimensión de autonomía y al hacerlo, el conocimiento deja de concebirse como una representación realizada por un sujeto pasivo. Por ende, entenderemos conocimiento como acción efectiva (Maturana y Varela, 1984), es decir, como un fenómeno incorporado y activo que involucra co-participativamente

\footnotetext{
${ }^{3}$ La noción de observador en este contexto de interpretación, se asocia más a la idea de participe que a la de espectador, en un reconocimiento implícito a los procesos de autonomía inherentes a toda observación.
} 
al observador y lo observado. De esta forma, el conocimiento será entendido, no como la representación de un mundo precedente al acto de distinción donde es configurado, sino más bien, como la construcción y configuración de un mundo basado en la historia de distinciones, que un observador es capaz de realizar como acciones efectivas en relación con su entorno. Enfatizando en las dinámicas auto-eco-organizadas de los sistemas cognitivos y parafraseando expresiones de Piaget, el observador, mediante sus patrones de conocimiento, organiza el mundo organizándose a sí mismo. ${ }^{4}$

Gradualmente las nociones generadas dentro del dominio de la epistemología constructivista han influenciado los modelos de interpretación y el entendimiento del fenómeno educativo, en lo referido a la trama de procesos de comunicación y aprendizajes. No obstante, es necesario indagar en qué dominios de la acción educativa se sitúan y toman forma las nociones implícitas a la epistemología constructivista, dado que los cambios de denotación no precisamente conllevan transformaciones profundas en los procesos de despliegue en el ámbito educativo, cualquiera sea su nivel o contexto.

La postura epistemológica constructivista supone según Glasersfeld (1996), un tránsito entre una postura ontológica basada en: a) un mundo completamente estructurado, preexistente e independiente de cualquier observador que lo experimente, y b) la tarea del observador de descubrir cómo es ese mundo "real" y su estructura; a una postura epistemológica que sostiene que: a) el conocimiento no se recibe pasivamente, ni a través de los sentidos, ni por medio de la comunicación, sino que es construido activamente por el sujeto cognoscente, y b) la cognición es adaptativa y sirve a la organización del mundo experiencial del sujeto, no al descubrimiento de una realidad ontológica objetiva." (Glasersfeld, 1996:24) En este sentido, la epistemología constructivista involucra la perspectiva sistémica, pero la expande al integrar una concepción que reconoce la relación analógica y circular que se da en la relación observador ßà observado, es decir, incorpora al observador en su observación.

En una perspectiva relacional y constructivista de la cognición, la operación de aislar un patrón desde una red de relaciones, dibuja una frontera arbitraria que define al objeto, en términos de relaciones preferenciales que se dan entre los diversos componentes que materializan dichas relaciones (Acevedo, 2000). Cabe enfatizar que esta operación de distinción está siempre mediada por la experiencia en todos sus órdenes de recursión. Así, las entidades o unidades en este sentido, son proyecciones y por ello están estrechamente relacionadas a las teorías y la cultura de quienes las proyectan (Feyerabend, 1990). En consecuencia, los sistemas cognitivos están imposibilitados de dar cuenta de una realidad independiente a ellos, lo que se traduce en una desontologización de la noción de realidad, en otras pala-

\footnotetext{
${ }^{4}$ Cabe enfatizar que la expresión inversa es igualmente pertinente desde un entendimiento auto-eco referencial de la cognición.
} 
bras, la lógica del mundo se transforma en la lógica de construcción de ese mundo (Arnold, 1997). Desde la síntesis propuesta por Bateson (1979), el nombre y la cosa nombrada, el mapa y el territorio, al corresponder a tipologías lógicas distintas, ya no son equivalentes y por ello no pueden ser confundidos. ${ }^{5}$ Derivado de este plano argumental, surge una concepción de ciencia que no explica lo real, sino campos fenomenológicos en base a un conocimiento contingente y constitutivo a la relación cognitiva en los cuales son configurados.

Dada la importancia de la noción de distinción en este contexto, la unidad organismo ßà entorno se hace indisociable. Para Lavanderos (2002), es posible entender el acto de conocer como un proceso de configuración de mundo y a su vez a la cultura, como la conservación de dichas formas particulares de configuración. De este modo, todo sujeto cognitivo es un configurador y el aprendizaje es, dentro de una red de comunicación, una instancia de conservación de dichas formas de distinción.

El origen del observador como unidad y su entorno, es co-circunstancial a la realización de la distinción, por lo que, observador y entorno son ontogenéticamente dependientes (Lahitte, Hurrell, Malpartida, 1989). Francisco Varela (2000: 241) señala al respecto "para que exista una mente tiene que haber manipulación e interacción activa con el mundo, entonces tenemos un fenómeno incorporado y activo, y cualquier cosa que denominemos un objeto, una cosa en el mundo, las sillas, las mesas, las personas y las caras y todo lo demás, depende totalmente de esta constante manipulación sensoriomotriz. No podemos captar al objeto como si simplemente estuviera "ahí afuera" en forma independiente. El objeto surge como fruto de nuestra actividad, por lo tanto, el objeto como la persona están co-emergiendo, co-surgiendo" .

De esta forma, la epistemología constructivista no se acota a una dimensión metodológica, ni a una metáfora arquitectónica del conocimiento, sino que más bien sobrepasa estas consideraciones, situándose como una emergente forma epistemológica de interpretar enactivamente la educación y todo su dominio de acción. Para von Foerster (1996), desde el instante en que el conocimiento generado involucra la responsabilidad del sujeto, la epistemología constructivista contiene la semilla de una ética.

Derivado de este plano argumental, aparece una concepción de observador como seleccionador activo de sus distinciones; esto es, según sostiene Morin (1994), un observador que percibe y además concibe en la relación cognitiva. De este modo, el énfasis en la autoorganización y la autonomía en los procesos biológicos y cognitivos, significa que un sistema auto-eco-poiético es a la vez un sistema cognitivo auto-exo-referente, que genera distinciones configurando y dando origen a un entorno y a él mismo como unidad.

\footnotetext{
${ }^{5}$ Esta idea supone distinguir sin desunir y simultáneamente, unir sin confundir.
} 
Rescatando la autonomía de los procesos vitales y cognitivos, Francisco Varela señala: “Organismos, fascinantes redes de sí mismos no sustanciales/vacíos, nada más ni nada menos que existencias circulares, de niveles múltiples y final abierto, siempre movidos por la falta de significación que ellos mismos engendran al afirmar su presencia”. (Varela, 2000:111). Al intentar conocer el conocer, nos encontramos nítidamente con nuestro propio ser (Maturana, Varela, 1984), en la indisoluble condición entre el conocer y el vivir. De la conjugación de las nociones de encarnación y emergencia, surge una concepción de mente y mundo intrínsecamente relacionada a la imaginación y fantasía ${ }^{6}$. El constructivismo, en este contexto, se presenta como una epistemología para educar en pluralidad o, como señalara Heinz von Foerster (1996), una epistemología para conocer y dejar conocer.

El fenómeno cognitivo no se basa tanto en la representación, denotación y/o descripción, sino en la co-generación cognitiva de lo que hemos aprendido a llamar realidad. La educación, en este contexto, no transmite explicaciones y descripciones de un mundo pre-dado, sino que genera y estabiliza constantemente esos mundos cognitivos mediante los esquemas de distinciones en los cuales sustenta sus explicaciones. Esta aproximación enfatiza que la educación es un proceso cognitivo que no sólo puede tratar con resultados o contenidos estandarizados, sino que genera/estabiliza las formas de conocer que amplían/restringen el horizonte de distinciones en las diversas relaciones que conforman la trama antropo-social a escala planetaria. Esto implica que el histórico rol adaptativo asumido por los sistemas educativos (análogo a un sistema espejo de lo social), ha sido basado en una trivial interpretación del fenómeno del conocer y sus formas de generación y comunicación (Oliva, 2007).

Con ello se refrenda la condición generativa y no sólo descriptiva/ denotativas de las explicaciones, posicionando a los procesos educativos en un nuevo estatus epistemológico, ya no centrado en la transmisión de mercancías y contenidos, sino en la generación de contextos cognitivos que posibilitan y constriñen los horizontes de distinciones ( $y$ de realidad) de una comunidad de observadores. Enfaticémoslo, la educación no trasmite ni transfiere ningún tipo de unidad cognitiva, sino más bien crea, legitima y consolida configuraciones de distinciones en dinámicas de carácter sistémicas y hologramáticas.

\footnotetext{
${ }^{6}$ Edgar Morin destaca la importancia del imaginario en el ser humano; enfatizando que las vías de entrada y de salida del sistema neuro-cerebral que conectan el organismo con el mundo exterior representan sólo el $2 \%$ de todo el conjunto, mientras que el 98\% implica al funcionamiento interior. Esto ha constituido un mundo psíquico relativamente independiente, donde se fermentan necesidades, sueños, deseos, ideas, imágenes, fantasmas, y este mundo se infiltra en nuestra visión o concepción del mundo exterior.
} 


\title{
Desde la abstracción a la situacionalidad
}

\author{
Amo la humanidad, lo que me empelota son las personas
}

Libertad (Mafalda)

Las dinámicas educativas no pueden enajenarse en patológicas abstracciones, no sólo es necesario el pensamiento crítico o denominado de orden superior, la reflexión crítica y la argumentación lógica, sino la vinculación afectiva con todos los órdenes de recursión de la experiencia. Para Morin (1999), la racionalidad dista de la racionalización, la primera abre mundos cognitivos, la segunda se clausura en la abstracción. La conformación de ismos ciegos e incapaces de contener la diversidad creativa, imposibilitan la integración profunda de la teoría y praxis en un campo de consecuencia y proyecciones éticas para la educación contemporánea.

Desde la perspectiva de Varela (1991, 2000), las concepciones centradas en el dominio abstracto de la mente son ampliadas desde una mirada dinámica, que reconoce en el fenómeno mental la importancia de la experiencia inmediata y moverse afectivamente en un mundo. Así, la inteligencia es mucho más fuerte cuando es tácita y activa (encarnada), cuando deviene de la manipulación sensoriomotriz en el entorno. Desde esta concepción, la mente no está en la cabeza sino que es inseparable del organismo como un todo, esto es, está encarnada en una co-determinación de lo interno y lo externo. En base al estado del arte en ciencias cognitivas, sujeto y objeto co-emergen en la relación, toda propiedad es co-emergente, por lo que la cognición está enactivamente encarnada.

De este modo, todo sistema racional se funda sobre un dominio constituido por premisas no racionales, por lo que el comprender es inseparable de la experiencia humana, ya que todo el reordenamiento racional cognitivo que pueda construirse, descansa en premisas tácitas que han sido proporcionadas por la experiencia inmediata (Maturana, 1990). Tal como señala von Foerster (1995), si bien debemos ver para creer, también requerimos creer para ver, en otras palabras, se debe comprender lo que ve, o de lo contrario no se le ve $\mathrm{v}^{7}$, por lo que las formas afectivo-lógicas de reformular los fenómenos (explicarlos) pueden facilitar o inhibir su distinción.

Se prescinde rescatar el valor del sentido común, la espontaneidad, la intuición y la inmediatez de la experiencia, desde la enajenada abstracción subyacente a corrientes tales como el denominado pensamiento críti$\mathrm{co}^{8}$. Francisco Varela (1991) ha advertido que la centralidad en lo abstrac-

\footnotetext{
${ }^{7}$ Supuesto de H. Von Foerster en base a los trabajos de Humberto Maturana y Sammy Frenk en la neurofisiología de las trayectorias visuales. Para una discusión más profunda referirse a Visión y conocimiento. Disfunciones de segundo orden, en Freíd, D. (1995) Nuevos paradigmas, cultura y subjetividad. Ed.Paidos Argentina.

${ }^{8}$ El llamado pensamiento crítico se consolida como un movimiento intelectual en Estados Unidos a finales de los años 60' desarrollando diferentes propuestas educativas y curriculares.
} 
to, a la luz de las ciencias cognitivas, debe replantearse hacia el reconocimiento de la historicidad y el contexto de una situacionalidad, esto es, nuestro mundo vivido está tan a la mano, que no tenemos un control acerca de lo que es y de cómo lo habitamos, por lo que siempre operamos en la inmediatez de una situación. Se trata aquí, de volver y recuperar la experiencia inmediata, o como señalara Edmund Husserl: "volver a las cosas mismas". Desde la perspectiva de Varela (1991), esto ha implicado que la ciencia cognitiva gradualmente comience a reconocer que el simple hecho de estar ahí, en la acción inmediata, dista mucho de ser un proceso "simple” o cuestión de "reflejos”, dado que además comportan procesos evolutivos mucho más amplios. Asimismo, Núñez (2004) sostiene que el sentido común y la inmediatez es la dimensión cognitiva más compleja en términos de un programa de investigación en ciencias cognitivas.

Los planos racionales y afectivos de la cognición son dominios inseparables, ya que ambos se originan en la experiencia fenomenológica del sujeto ßà cultura, proceso que siempre opera como una totalidad irreductible a sus parcialidades. En este contexto, la experiencia (plano observacional) y la reformulación de la experiencia (plano argumental) corresponden a dominios diversos pero convergentes, distinguibles pero no disociables. En este devenir, la aparición del lenguaje y la emergencia del espacio simbólico de lo humano, posibilita un segundo orden de distinciones, transitando desde un dominio de experiencia inmediata, a un dominio racional o argumentativo. Ambas dimensiones conforman el complejo bucle epistemológico desde el cual surgen las diversas dimensiones cognitivas de lo humano, en un constante proceso de co-producción fenomenológica.

En la experiencia es posible describir sucesivos niveles de recursión que, como tales, no permiten distinguir planos de origen o término, sino que se confunden en una trama experiencial indisociable. Ahora bien, al margen de los “ $n$ ” órdenes de recursión de los que pueda estar conformada la experiencia humana, genera un solo y complejo fenómeno, a saber, la experiencia fenomenológica. Tal como formula Varela (2000: 108) ${ }^{9}$ : “El organismo como tal, entonces, no puede ser abordado como un proceso único. Nos vemos forzados a describir "regiones" que se entretejen de complejas maneras y, en el caso de los humanos, se extiende más allá de los estrictos límites del cuerpo hasta el registro socio-lingüístico”. Simultáneamente, la experiencia sobrepasa cualquier intento de reformulación, es decir, la cantidad, diversidad y convergencia de distinciones que agitan a un sujeto en su experiencia, no son susceptibles de reformular cabalmente en un plano argumental, en palabras de Goethe: "la experiencia es siempre

Esta corriente enfatiza en la potenciación de la educación del pensamiento de orden superior, la reflexión crítica y la argumentación lógica.

${ }^{9}$ Este artículo, originalmente publicado en 1991 como Organism: A meschwork of selfless selves, en Tauber (ed), Organism and the origin of self, Kluwer Assoc, Dordrecht, pp.79107, forma parte de la compilación de la obra de Francisco Varela El fenómeno de la vida (2000). 
sólo la mitad de la experiencia” (en Dörr 1996:11). Derivado de ello, el campo experiencial sobrepasa cualquier intento de reformulación a un campo argumentativo o racional y es por ello que se mantiene en una constante fuga.

No obstante, una reformulación o argumentación, es una configuración de distinciones de órdenes secundarios y, como tal, puede a su vez reformular y transformar la experiencia inmediata que deviene de ella. Teoría y praxis se encuentran fusionadas en la experiencia compleja del observador, por lo que toda perturbación en cualquiera de sus órdenes o dimensiones, puede transformarla en su ámbito global. Podemos aprender a reconocer la importancia de la alteridad y presencia del otro (ya sea humano o no humano), desde planos experienciales inmediatos, por ejemplo cualquier dinámica relacional de acogida, como a su vez, desde campos abstractos y reflexivos, por ejemplo, una teoría biológica o antropológica de la alteridad. Quizás convengamos que aunque fundamental, este segundo campo fenomenológico nunca logrará reemplazar al primero.

Para Bateson (1972) ningún organismo puede darse el lujo de hacer consciente, procesos que puede manejar a nivel inconsciente. Sin embargo, la experiencia humana ya no puede prescindir de la conciencia, está constituida en ella, por lo que la consciencia de la consciencia, el conocer del conocer son tareas transversales a todo proceso educativo. William Blake lo señalaba en su poesía cuando expresaba que: "una lágrima es a la vez un fenómeno intelectual”. A la luz de ello, la clásica disyunción teoría-praxis parece entonces una gran quimera.

Existe una diferencia capital entre explicar una cosa o evento (lo que evidentemente es una esquizofrenia) y vivir la explicación como una instancia convergente y constitutiva de la experiencia; esto es, explicar implica reformular los mismos campos fenomenológicos en los que estamos imbricados. En este sentido el mayor acto de rigor al que podría aspirar la actividad científica es la consecuencia e integración del observador en su observación.

Desde Aristóteles, hemos aprendido a maravillarnos de los procesos de emergencia y como el todo, cualitativamente, se hace mayor que la sumas de sus partes. No obstante, los procesos de constricción también son inherentes a toda relacionalidad cognitiva local-global. La actividad cognitiva, con su juego de niveles de recursión, posibilitó la producción de esa noosfera semiótica que llamamos cultura y que conforma nuestras tramas de existencia. En ella la humanidad ha creado la historia, música, literatura, arte, filosofía, poesía, etc., ampliando cada vez más nuestros dominios fenomenológicos de experiencias; sin embargo, y simultáneamente a ello, la cultura nos restringe, nos coarta, masifica y trivializa. La cultura (y holográficamente la educación), a medida que abre, constriñe la experiencia humana.

En el seno de lo humano es costumbre vanagloriarse de los $n$ niveles de abstracción que ha generado la actividad cognitiva humana. Con un sentido casi romántico nos referimos al pensamiento humano y su belleza, como 
si los procesos racionales fueran garantes de estados de conciencia cada vez más complejos. Es posible postular que a mayores grados de recursión en los procesos de distinción se dan mayores grados de abstracción de la experiencia, no obstante, si estos procesos no vuelven a su origen, se transformar en argumentaciones auto-referentes que fracturan las vías ecoreferenciales a los dominios sensibles que las posibilitan. Con ello, las teorías y las ideas se clausuran en un racionalismo que poco tiene que ver con la racionalidad y sus dominios de apertura de la experiencia humana. Todo proceso intelectual arriesga un cierre o clausura racional que lo hace ciego a sus orígenes, imposibilitando estados de conciencia convergentes. Los niveles de conciencia no se alcanzan solamente por abstracción y sofisticación sino, insistamos, por consecuencia.

Una reforma de la educación no es posible tal como advierte Motta (1999) sin una reforma del pensamiento, cuyos primeros pasos implican una ecología de la inteligencia colectiva y una ecología de las ideas. No obstante advierte: ¿ es posible una reforma del pensamiento sin el despertar de una nueva sensibilidad? Y esa nueva sensibilidad ¿podrá emerger en la banalidad y el comentario nihilista en que están encerrados el arte, la política, el amor, el pensamiento y la religión?

En síntesis, el dominio abstracto abierto por las posibilidades recursivas (reflexivas) del lenguaje es un dominio constituyente y trasformador de la experiencia, pero que no la reduce a él. Si bien el conocimiento debe abrirse (y no clausurarse) hacia órdenes superiores de reformulación de la experiencia (reflexión, crítica, razonamiento creativo, argumentación lógica), es igualmente necesario volver a situar la experiencia inmediata como fundadora de todo el complejo proceso cognitivo. No hacerlo, como advierte Merleau-Ponty (1997) implicaría una reflexión incompleta que carecería de la conciencia de su propio comienzo. Por lo tanto, la dimensión epistemológica comportaría la comprensión que todo proceso educativo (formal e informal) se desarrolla en el interior de un bucle conformado por la situacionalidad/inmediatez/sensibilidad y lo intencional/lógico/abstracto.

\title{
Educación y complejidad
}

\author{
El universo está hecho de unión \\ El universo es condensación \\ Condensación es unión y es calor. (Amor) \\ El universo es amor
}

\section{E. Cardenal}

A la luz de la convergencia de los tres apartados anteriores, los aportes de la epistemología de la complejidad contribuyen con un dominio de entendimiento para abordar la dimensión paradigmática y ética del fenómeno educativo. Es por tanto, en esta perspectiva, una respuesta relevante para la orientación de estrategias que tiendan a superar la enajenación en la 
cuantificación, sustantivación, denotación y abstracción del conocer en educación.

Desde esta referencia epistemológica, es plausible aproximarse a una noción compleja del fenómeno educativo, donde adquiere una dimensión procesual y sistémica que enfatiza en procesos de co-creación relacional y no en transferencias de mercancías cognitivas. En el sentido de Bateson (1972) la epistemología aborda por una parte, los procesos de conocimiento, pensamiento y decisión y, por otra, los límites necesarios y otras características de dichos procesos. Los sistemas cognitivos, conocen ßà actúan según los paradigmas generados holográficamente por la educación y la cultura, en las formas en que aprendieron a configurar mundo. De este modo, al dominio epistemológico no sólo le conciernen las modalidades analógicas de configuración que operan en las formas de conocimiento, sino también la acción que sobreviene de él, es decir, cómo conocemos y cómo educamos. Para Morin (1994) aun cuando la dimensión cognitiva se diferencie y autonomice, sigue siendo inseparable de la acción; ésta conforma un bucle ser-hacer-conocer.

La reinterpretación a nivel epistemológico del fenómeno educativo, necesariamente trasunta en una transformación de las nociones asociadas a su definición y en los programas de políticas públicas que devienen de ellas. De esta forma, la noción de calidad educativa y los programas de acción desarrollados, justificados y legitimados en este contexto, deben revisarse a la luz de emergentes formas epistemológicas más complejas de interpretar la educación y el fenómeno del conocer. La trivialización, simplificación y mercantilización del conocer desde la base de sustratos epistemológicos falaces, comporta el riesgo de restringir los sentidos de la educación bajo esquemas sumativos, transmisionistas, constrictivos y reproductivos. Las corrupciones epistémicas que infiltran los espacios educativos configuran miedos, frustración y desidia, convirtiendo un fenómeno complejo y multidimensional en una quimera que se nutre de linealidad, determinismo y trivialidad. En síntesis, una reforma educativa de orden no trivial como la esbozada, conlleva una transformación meta-política que es, a la vez, una revolución epistemológica. 


\section{Bibliografía}

Acevedo, R. (2000), La noción de sistema bajo el enfoque de las ontologías constitutivas. Edición UTFSM. Valparaíso. Chile

Bateson, G. (1972), Steps to an ecology of mind. Ballantine Books / Random House, New York. EE.UU.

Dörr, O. (1996), Espacio y tiempo vividos: Estudios de antropología psiquiátrica. Ed.Universitaria. Santiago de Chile.

Maturana, H. (1990), Emociones y lenguaje en educación y política. CED Santiago de Chile.

Merleau-Ponty, M. (1997), Fenomenología de la percepción. Península. Barcelona, España.

Morin, E. (1992), El método. Las ideas. Cátedra, Madrid, España.

Ídem (1994), El método: El conocimiento del conocimiento. Ed. Cátedra, Madrid, España.

Ídem (1999), Los siete saberes de la educación del futuro. UNESCO, Francia.

Ídem (2001), Introducción al pensamiento complejo. Gedisa, Barcelona, España

Motta, R. (2002), “Complejidad, Educación y Transdisciplinariedad”. Revista Polis Académica. Universidad Bolivariana. Volumen 1 Número 3. [Documento en Línea]. http:// revistapolis.cl/3/motta3.doc.

Núñez, R. (2004), "El paradigma de la mente corporizada”. En Fischer, A. (Editor) Nuevos paradigmas a comienzos del tercer milenio. Ediciones Aguilar. Santiago de Chile.

Oliva, I. (2007), “Aspectos contextuales y epistemológicos para una transformación educativa”. Complexus. Ciencia, arte y estética. Vol.3 №1. Pag. 87-97

Varela, F. (1991), Ética y acción. Dolmen Ensayos, Santiago de Chile.

Ídem (2000), El fenómeno de la vida. Dolmen Ensayos, Santiago de Chile.

Von Glaserfeld, E. (1996), “Aspectos del constructivismo radical”. En Pakman, M. (comp.) Construcciones de la experiencia humana. Vol. 1. Ed.Gedisa. Barcelona, España. 
Von Foerster, H. (1995), "Visión y conocimiento. Disfunciones de segundo orden”. En D.Freíd Nuevos paradigmas, cultura y subjetividad. Ed.Paidos Argentina.

Ídem (1996), Las semillas de la cibernética. Edición de M.Pakman. Editorial Gedisa. España. 